\title{
Seeing Invisible Light: 2-photon microperimetry to measure visual function
}

Urmi Mehta ${ }^{\mathrm{a}, \mathrm{b}}$, Grazyna Palczewska ${ }^{\mathrm{a}, \mathrm{c}}$, Ken Y. Lin ${ }^{\mathrm{a}}$, Andrew W. Browne $\mathrm{e}^{\mathrm{a}, \mathrm{d}, \mathrm{e}}$

a. Gavin Herbert Eye Institute, Department of Ophthalmology, University of California-Irvine, Irvine, CA 92617, USA

b. Western University of Health Sciences, Pomona, CA 91766, USA

c. Polgenix Inc., Department of Medical Devices, Cleveland OH 44106

d. Institute for Clinical and Translational Sciences, University of California-Irvine, Irvine, CA 92617, USA

e. Department of Biomedical Engineering, University of California-Irvine, Irvine, CA 92617, USA

\section{Corresponding Author:}

Urmi Mehta, BS

University of California-Irvine School of Medicine

850 Health Sciences Rd,

Irvine CA 92697

714-656-5050

mehtau@uci.edu 


\begin{abstract}
Purpose: The accuracy of conventional visual function tests, which emit visible light, decreases in patients with corneal scars, cataracts, and vitreous hemorrhages. In contrast, infrared (IR) light exhibits greater tissue penetrance than visible light and is less susceptible to optical opacities. We therefore compared visual function results obtained using conventional visual function tests and infrared 2-photon microperimetry (2PM-IR) in a subject with a brunescent nuclear sclerotic and posterior subcapsular cataract before and after cataract surgery.

Methods: Visual function testing using the cone contrast threshold (CCT) test, conventional microperimetry (cMP), visible light microperimetry from a novel device (2PM-Vis), and 2PMIR were performed before and after cataract surgery.

Results: Cone contrast threshold testing improved for the S-cone, M-cone, and L-cone by 111, 14, and 30. Retinal sensitivity assessed using cMP, 2PM-Vis, and 2PM-IR improved by $18 \mathrm{~dB}$, $17.4 \mathrm{~dB}$, and $3.4 \mathrm{~dB}$, respectively.

Conclusions and Importance: 2PM-IR, unlike conventional visual function tests, showed minimal variability in retinal sensitivity before and after surgery. Thus, IR visual stimulation introduces a paradigm shift for measuring visual function in the retina and posterior visual pathways by circumventing optical media opacities.
\end{abstract}

\title{
Keywords:
}

Two-photon microperimetry, cataracts, visual sensitivity 


\section{Introduction:}

Cataracts are the leading cause of reversible vision loss worldwide ${ }^{1}$. By attenuating (scattering) light entering the eye, cataracts reduce visual acuity, color vision, and contrast sensitivity. Patients may also report increased glare and visual field $\operatorname{loss}^{2}$. Eye care providers rely upon visual function tests to measure changes in vision. However, visual function test performance is confounded by media opacities ${ }^{2-5}$ like cataracts. Cataracts, an age-related condition, coincide with other common age-related diseases such as age-related macular degeneration (AMD) and glaucoma. Precision in isolating and quantifying neurosensory retinal function is therefore diminished by cataracts. In fact, no visual function test exists that can distinguish the earliest stages of AMD from normal age-matched eyes ${ }^{6}$.

IR light has greater transmittance into the aged eye than visible light ${ }^{7}$. Two-photon (2P) technologies prevail in the basic sciences where conventional fluorescence microscopy using short wavelength light to stimulate fluorescence has been partially supplanted by $2 \mathrm{P}$ microscopes in which ultrafast pulsed infrared lasers introduce 2 photons of infrared light to a small focal volume to stimulate fluorescence much like conventional fluorescence microscopes using UV and visible light. Recent innovations in 2P technology have demonstrated that pulsed IR light can initiate phototransduction by inducing photoisomerization of 11 -cis-retinylidene ${ }^{8}$ nested in visual pigments. Therefore, 2P IR light can stimulate human vision.

While IR light has long enabled medical imaging of the ocular fundus, this report demonstrates the inaugural clinical use of 2PM-IR in a 51-year-old male with a brunescent nuclear sclerotic (NSC) and posterior subcapsular cataract (PSC) before and after cataract surgery. 


\section{Materials and Methods:}

This study received Institutional Review Board approval from the University of California, Irvine and was conducted in accordance with the Declaration of Helsinki and HIPAA regulations (IRB protocol number 2018-4603). Informed written consent was obtained from the patient.

\section{Baseline Evaluation}

A 51-year-old Caucasian male presented to the Gavin Herbert Eye Institute with gradual painless vision loss of the left eye over the preceding 2 months with a reduction in best corrected visual acuity (BCVA) at distance from 20/25 to 20/70. Slit lamp exam showed a dense brunescent and posterior subcapsular cataract (Fig. 1). Medical history revealed a branch retinal arterial occlusion of the left eye at the age of 19 and borderline glaucoma. The patient was subjectively dependent on his left eye after losing vision in his right eye secondary to CMV retinitis with a stable pseudophakic vision of 20/40. There was no history of prior surgeries or trauma to the left eye.

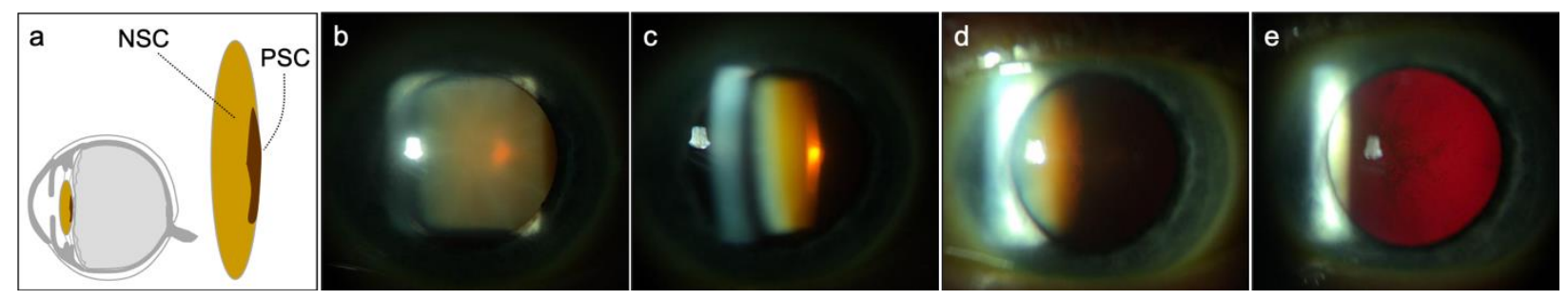

Figure 1. Diagram of cataract with NSC and PSC (a). Brunescent nuclear sclerosis is highlighted in vivo by broad illumination (b), and slit beam illumination of the lens nucleus (c). PSC is revealed by slit beam illumination of the lens posterior capsule (d), and retroillumination (e). 
Given his monocular vision status, he was scheduled for expedited cataract surgery using a clear monofocal intraocular lens (ZCB00, Abbott Medical OpticsSanta Ana, CA) during the COVID19 pandemic.

\section{Functional Testing}

Color vision and retinal sensitivity were assessed before and 1 week after cataract surgery using a battery of conventional visual function tests and 2PM-IR. Cone contrast threshold (CCT) testing was employed to measure color and contrast vision (ColorDx CCT HD, Konan Medical Irvine CA) under photopic conditions. Focal retinal sensitivity at multiple points was measured under photopic conditions using a conventional microperimeter (cMP, Nidek MP-3, San Jose, CA). The 2-photon microperimeter (2PM) can present either a visible light green stimulus (2PMVis) at $522.5 \mathrm{~nm}$ or it can stimulate vision using a $1045 \mathrm{~nm}$ pulsed infrared light source (2PMIR) under scotopic conditions. The average from 5 measurements was calculated for 2PM-IR and 2PM-Vis. Color fundus photographs (CFP, Clarus 500, Karl Zeiss Meditec, Dublin CA) were taken and assessed for change in image characteristics.

\section{Results:}

BCVA at distance was 20/25 at the patient's 1-week post-op visit. Improvement in CCT scores and retinal sensitivity before and 1 week after cataract surgery are shown in figure 2.

CCT scores for 3 cone classes and retinal sensitivities measured on three microperimetry devices demonstrated a statistically significant improvement after cataract surgery. Retinal sensitivity 
improved by a similar amount for both visible spectrum microperimetry assays, with $18 \mathrm{~dB}$ for conventional microperimetry and $17.4 \mathrm{~dB}$ for 2PM-Vis. 2PM-IR demonstrated a $3.4 \mathrm{~dB}$ change in visual sensitivity, which is approximately $20 \%$ of the change seen with visible spectrum microperimetry. Preop and postop standard deviations were $1.9 \mathrm{~dB}$ and $0.7 \mathrm{~dB}$ for $2 \mathrm{PM}$-Vis and $0.4 \mathrm{~dB}$ and $0.7 \mathrm{~dB}$ for $2 \mathrm{PM}-\mathrm{IR}$, respectively.

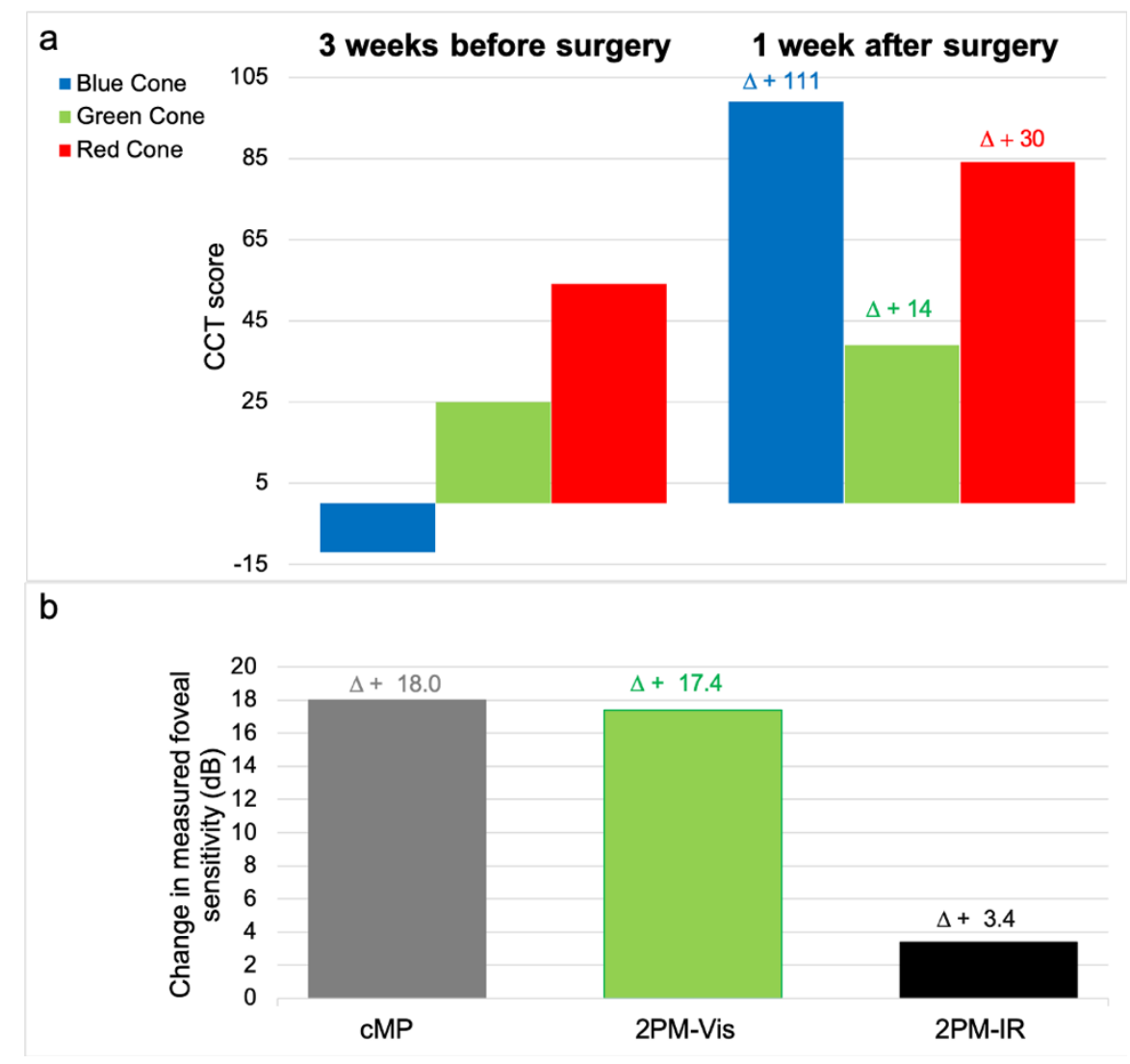

Figure 2. Changes in visual function testing before and after cataract surgery. CCT scores before and after cataract surgery for the cone contrast threshold (CCT) test (a) and change in foveal sensitivity for three different microperimetry assays (b) are plotted as bar graphs. 
Optical imaging of the retinal fundus demonstrates the ability for light outside the eye to pass through the optical media, reflect on the retinal fundus, and return through the optical media to the camera sensor (Fig. 3). Color fundus photographs are qualitatively redder and blurrier before surgery. Colorimetry (ImageJ) of the optic nerve head, the whitest part of the fundus, demonstrates the greatest change in number of image pixels for blue pixels, followed by green. Red pixel count didn't change in fundus photos after cataract surgery. Infrared imaging at two wavelengths (750 and $840 \mathrm{~nm}$ ) demonstrated retained image features when features in color photography were lost in the context of PSC (central column of Fig. 3). All imaging modalities demonstrate improved image clarity after cataract surgery. 


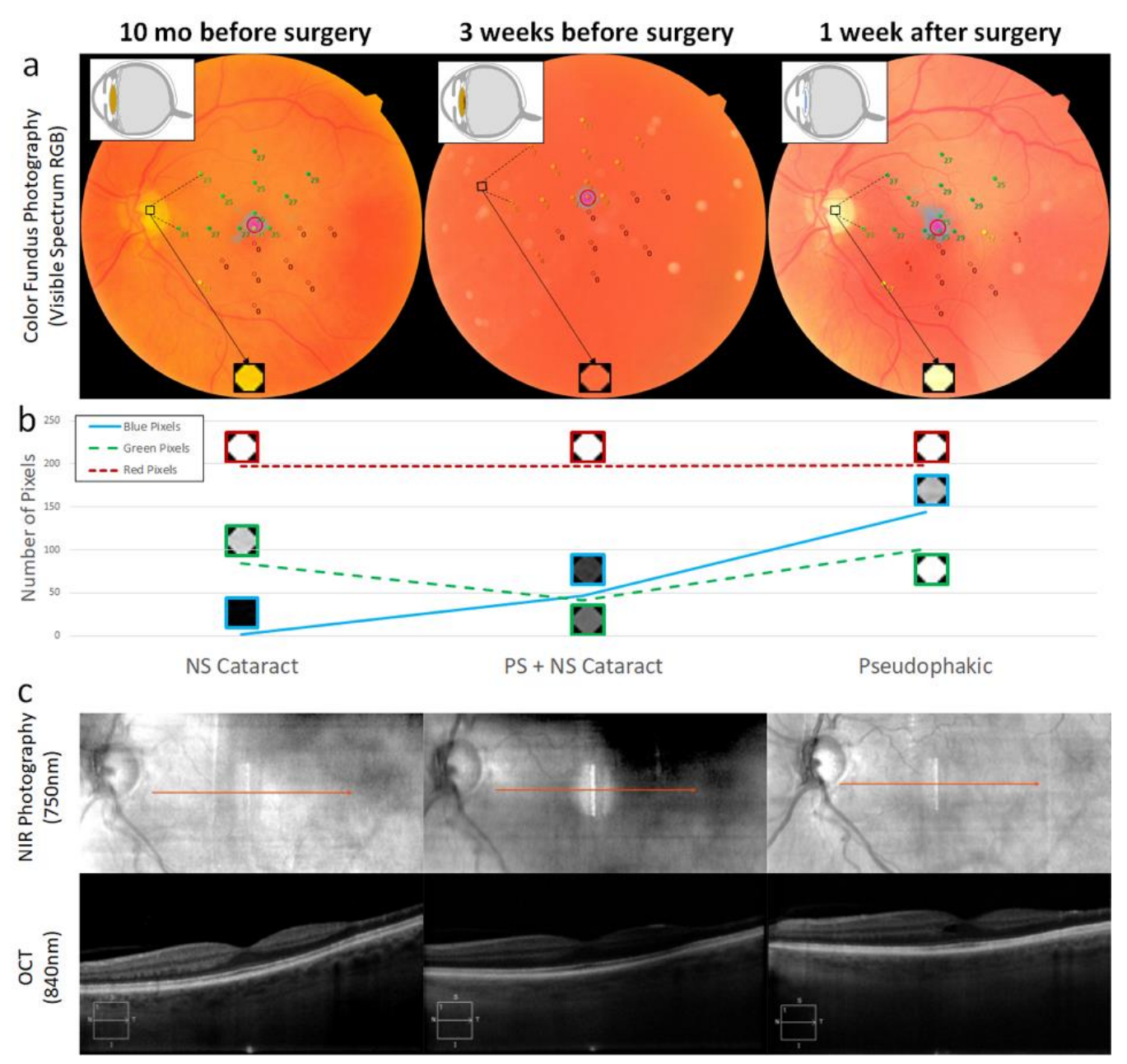

Figure 3. Changes in multimodal imaging acquired with NSC alone 10-months prior to surgery, with both NSC and PSC 3-weeks prior to surgery and pseudophakia 1-week after cataract surgery. (a) Color fundus photographs (visible spectrum composed of red, green and blue channels). (b) Quantitative analysis of visible spectrum images of the optic nerve head reveals changes in total blue, green and red pixels at each timepoint. Insets (bottom center of color fundus photographs) are the sampled image area from the optic nerve head which were separated and analyzed for each blue, green and red color channel (color channel-specific sample is overlaid onto the graph in b). (c) Infrared imaging by NIR fundus photography $(750 \mathrm{~nm})$ and 
OCT b-scan $(840 \mathrm{~nm})$ demonstrate preserved visible anatomy at all stages of cataract. Nuclear sclerotic cataract (NSC); posterior subcapsular cataract (PSC); near infrared (NIR); optical coherence tomography (OCT).

\section{Discussion:}

Our case report demonstrates that limitations in conventional visual function tests arising from media opacities can be overcome by 2PM-IR. This is the first report using 2PM-IR in vivo that shows minimal changes in retinal sensitivity after the removal of a visually significant cataract. Our patient presented with a brunescent nuclear sclerotic and posterior subcapsular cataract of the left eye. Conventional visual function testing and color fundus photography before and after cataract surgery were largely dependent upon the presence or absence of media opacities. Prior studies by Ruminski et al comparing 2PM-Vis and 2PM-IR performance using human donor lenses found decreased absorbance and increased transmission of IR light ${ }^{8}$. This report corroborates their findings in vivo by demonstrating less variability in retinal sensitivity testing before and after cataract surgery using 2PM-IR than conventional visible spectrum assays. This study highlights the limitations of conventional visual function tests used in all clinical trials to accurately measure retinal function in the presence of a media opacity.

\section{Conclusion:}

The ability to diagnose and monitor function of the neurosensory retina in the presence of media opacities can be achieved with greater reliability and accuracy by using IR light. 2PM-IR may emerge as an adjunct to conventional vision testing to mitigate the effect of media opacity and help discriminate heretofore indistinguishable changes in visual function in early common blinding diseases like AMD. 


\section{Patient Consent:}

The patient consented to publication of the case in writing and orally.

Acknowledgements and Disclosures:

Funding: RPB unrestricted grant to UCI Department of Ophthalmology; ICTS KL2 Grant number is KL2 TR001416

Conflicts of Interest: Grazyna Palczewska is an employee of Polgenix, Inc. The following authors have no financial disclosures: UM, KYN, AWB

Authorship: All authors attest that they meet the current ICMJE criteria for Authorship.

Acknowledgements: The authors would like to thank Dr. Krzysztof Palscewski for use of the 2PM. The authors would also like to thank Carl Zeiss Meditec for loan of ophthalmic testing devices used to verify ophthalmic health in human subjects and Konan medical for an unrestricted donation of ColorDX. 


\section{Resources:}

1. Lam D, Rao SK, Ratra V, et al. Cataract. Nat Rev Dis Primers. 2015;1:15014.

2. Brown NA. The morphology of cataract and visual performance. Eye (Lond). 1993;7 ( Pt 1):63-67.

3. Fujikawa M, Muraki S, Niwa Y, Ohji M. Evaluation of clinical validity of the Rabin cone contrast test in normal phakic or pseudophakic eyes and severely dichromatic eyes. Acta Ophthalmol. 2018;96(2):e164-e167.

4. Palkovits S, Hirnschall N, Georgiev S, Leisser C, Findl O. Effect of cataract extraction on retinal sensitivity measurements. Ophthalmic Res. 2020.

5. Richter-Mueksch S, Sacu S, Weingessel B, Vecsei-Marlovits VP, Schmidt-Erfurth U. The influence of cortical, nuclear, subcortical posterior, and mixed cataract on the results of microperimetry. Eye (Lond). 2011;25(10):1317-1321.

6. Cocce KJ, Stinnett SS, Luhmann UFO, et al. Visual Function Metrics in Early and Intermediate Dry Age-related Macular Degeneration for Use as Clinical Trial Endpoints. Am J Ophthalmol. 2018;189:127-138.

7. Boettner EA, Wolter JR. Transmission of the Ocular Media. Investigative Ophthalmology \& Visual Science. 1962;1(6):776-783.

8. Ruminski D, Palczewska G, Nowakowski M, et al. Two-photon microperimetry: sensitivity of human photoreceptors to infrared light. Biomedical optics express. 2019;10(9):4551-4567. 